\title{
Spectral analyses of activity of laryngeal and orofacial muscles in stutterers
}

\author{
Anne Smith, Erich Luschei, Margaret Denny, Jennifer Wood, Minoru Hirano, \\ Steven Badylak
}

\begin{abstract}
Previous studies have reported that the disfluent speech of stutterers is often associated with tremor in orofacial muscle systems. In the present report, spectral analyses of the amplitude envelopes of laryngeal and orofacial EMGs revealed that tremor-like oscillations of EMG activity, similar to those observed in orofacial muscles, are also present in laryngeal muscles during stuttered speech. Furthermore, tremor-like oscillations in orofacial and laryngeal muscles appear to be entrained in some subjects. It is speculated that autonomic systems may provide a mechanism whereby oscillations in different muscle groups may become entrained.
\end{abstract}

(F Neurol Neurosurg Psychiatry 1993;56:1303-1311)

Stuttering is a disorder in which the motor outflow to the motor neuron pools recruited for speaking fails to generate normal patterns of speech movements. The precise aetiology of this disorder is unknown, but recent multifactorial models implicate genetic, psychosocial, physiological, linguistic, and other factors as significant in the development of stuttering. ${ }^{1-3}$ To understand the fundamental nature of this disorder and to develop successful therapeutic techniques, it would be important to specify the sources in the nervous system that are responsible for the failure of the motor command signals in stutterers' speech. Hypotheses about sources of inputs to motor neuron pools in stuttering must be based on precise descriptions of what actually is aberrant about muscle activity in stuttering.

A number of investigations from our laboratory and others have provided convincing evidence that speech breakdowns in stuttering (disfluencies) are often characterised by abnormal oscillations of EMG activity in muscles of the jaw, lips, and neck. ${ }^{4-6}$ These oscillations of EMG typically occur in a frequency band of 5-15 Hz. To the extent that tremor has been studied in orofacial systems, it appears that this frequency band overlaps that of normal physiological tremor. ${ }^{78}$ On the basis of such observations, it has been suggested that part of the disruptive drive to motor neuron pools in stuttering originates within the central and peripheral neural pathways that generate normal tremor. ${ }^{346}$

To evaluate the relative importance of reflex, central, and biomechanical factors in the generation of tremor 9 an important experimental step is to determine if oscillations occur at common frequencies across effector systems. For example, Phillipbar et $a l^{10}$ found that tremor in eight patients with Parkinson's disease did not show common spectral characteristics across limb, jaw, and vocal systems. This finding argues against a strong central component driving the oscillations in various effectors. Smith ${ }^{6}$ found that spectra of amplitude envelopes of EMGs recorded from jaw, lip, and neck muscles could show common frequencies of dominant oscillation during intervals of stuttered speech. Denny and Smith ${ }^{11}$ observed similar results in orofacial muscles during stuttered speech and reported that oscillations were highly correlated across muscles in some subjects. Denny and Smith also reported that oscillations in the $5-15 \mathrm{~Hz}$ band were not present in the fluent speech of these subjects. Thus, current evidence from recordings of orofacial muscles suggests that tremor-like oscillations grow large during disfluent intervals, but that abnormally high levels of oscillatory activity are not present during the fluent speech intervals of stutterers. Furthermore, in some stutterers, oscillations at a single frequency can be found in muscles of different articulators (for example, jaw and lip), and the amplitude of these oscillations may be correlated across muscles over time.

The results reviewed above describe abnormal characteristics of activity of orofacial muscles during stuttering. An obvious question is whether other systems involved in speaking, laryngeal and respiratory systems, are disturbed in similar ways in stuttering. Thus, the focus of the present investigation is the analysis of activity of intrinsic laryngeal muscles during stuttering.

Despite the important role often ascribed to laryngeal function in stuttering, only two investigations have been reported in which activity of intrinsic laryngeal muscles was recorded during stuttering. ${ }^{1213}$ Neither study reported any qualitative or quantitative description of oscillations of laryngeal muscle activity associated with stuttering.

Tremor has often been studied by computing the spectrum of the amplitude envelope of the rectified, low pass filtered EMG (for example, the "demodulated EMG" of Elble and Randall $\left.{ }^{14}\right)$. Using this analysis technique, the present investigation was designed to determine (1) the normal spectral composition of the EMG envelope of laryngeal mus- 
cles during speech and other vocal tasks, (2) if stuttered speech is associated with abnormal dominant oscillations of laryngeal EMG, and (3) if oscillations of laryngeal and orofacial muscle activity are correlated.

\section{Method}

SUBJECTS

Ten adult subjects were tested. Subjects N1N3 were control subjects, two women aged 22 and 38, and one man aged 50. These three subjects exhibited normal speech with a mean speech rate of 147 words/min $(S D=$ 15).

S1-S7 were stutterers with a history of stuttering onset in childhood. Mean speech rate for the seven stuttering subjects, including disfluencies, was 77 words $/ \mathrm{min}(\mathrm{SD}=25)$, and the mean number of disfluencies $/ 100$ words was $32(S D=11)$. On the basis of standardised measures, ${ }^{15}$ stuttering subjects were rated by a speech language pathologist as severe (five subjects) or moderate (two subjects). In view of the invasive nature of the experimental procedures, mild stutterers were excluded from the subject population. Previous experience has shown that under similar experimental conditions, mild stutterers often do not have enough disfluencies on which to perform the data analysis. Subjects N1-N3 and S1-S4 were tested at the Purdue speech physiology laboratory; data for S5-S7 were provided by Dr Christy Ludlow of the National Institutes of Health's (NIH) voice and speech laboratory.

\section{DATA COLLECTION AND EXPERIMENTAL TASKS} (N1-N3 AND S1-S4)

It was our goal to record the activity of two intrinsic laryngeal muscles, left thyroarytenoid (L-TA) and left cricothyroid (L-CT), and two orofacial muscles, left orbicularis oris inferior (L-OOI) and right levator labii superior (R-LLS). Analyses were planned for the two laryngeal recordings and for one channel of orofacial EMG.

For laryngeal recordings, electrodes were inserted into intrinsic laryngeal muscles with a 27 gauge hypodermic needle by an otolaryngologist with extensive experience with laryngeal EMG. The bipolar hooked wire electrodes consisted of two 0.002 inch diameter stainless steel wires with insulation removed from approximately $2 \mathrm{~mm}$ at the ends of the hooks. Laryngeal insertions were made according to the procedures described in detail by Hirano. ${ }^{16}$ These procedures included local anaesthesia of the skin at the insertion site with $1 \%$ lidocaine.

Successful placement of electrodes in TA or CT was assessed according to standard verification gestures for each muscle. ${ }^{16}$ If electrode insertion did not result in successful placement according to the experimenters' on line evaluation of patterns of EMG activation for the verification gestures, electrodes were removed. If the subject felt that he/she could easily tolerate another attempt, insertion was repeated on the same or the opposite side.
The activity of orofacial muscles was recorded with surface electrodes taped to the skin overlying the muscle.

All EMG and other signals were recorded on FM tape (bandpass $0-1250 \mathrm{~Hz}$ ) for off line analyses. Other signals recorded simultaneously with the EMGs were the subject's voice and movement of the lower lip. The latter signal was transduced with a strain gauge cantilever attached to the vermilion border of the lower lip at midline. ${ }^{17}$ The audio and movement signals were not analysed, but served as aids to determine the time at which samples of EMG during fluent and disfluent speech and other experimental tasks should be extracted for analysis. In addition, a video recording of the subject (including face and torso) was obtained.

After verification of the EMG recordings, subjects were asked to perform pitch glides by singing from low pitch to high pitch and from high to low pitch. They were then asked to phonate the vowel "ee" (as in beet) as long as possible. Performance of each of these tasks was repeated several times. Subjects then engaged in conversational speech and read passages for approximately 30 minutes of continuous data collection. The conversations covered topics such as the subjects' history of stuttering, their employment, their hobbies, etc. Subjects also read several passages, including two passages from an anatomy text that included difficult vocabulary. At the end of the speech and reading tasks, subjects repeated the verification gestures so that the experimenters could determine if the laryngeal electrodes had been dislodged.

\section{DATA COLLECTION AND EXPERIMENTAL TASKS} (S5-S7)

Data were collected for S5, S6, and S7 at the NIH voice and speech section in an extensive protocol designed to provide baseline measures of laryngeal function before treatment by injection of botulinum toxin into the vocal folds. The protocol included a short speech sample that, for severe stutterers, contained enough disfluent speech for the present analysis. Because the goals of the NIH experiment were different, the data channels available were not identical to those recorded from the subjects at the Purdue facility, but the data included both orofacial and intrinsic laryngeal muscle recordings.

Methods employed at the NIH laboratory for laryngeal and orofacial EMG recording were essentially identical to those employed at the Purdue facility. Data were provided on FM tapes recorded with the same model recorder operated at the same speed as that used for the Purdue data collection.

\section{DATA ANALYSIS}

For each subject three channels of EMG and the audio signal were digitised on a laboratory computer. When possible, the EMGs selected were two laryngeal EMGs and an orofacial EMG showing consistent activity. The sampling rate was 2048 samples/second/channel. 
Before digitisation the EMG signals were filtered, bandpass $50-1000 \mathrm{~Hz}$ for the laryngeal and $0-1000 \mathrm{~Hz}$ for the orofacial EMGs.

For each task performed by each subject, an attempt was made to digitise 20 intervals of 1 second duration. These intervals were taken for four conditions designated as:

FLU-the perceptually fluent speech of normal speakers and stutterers

STUT-the perceptually stuttered speech of stutterers

GLIDE- the pitch ascending and descending tasks

"ee"-the sustained vowel

Recorded muscles were not consistent across all subjects owing to failure to place electrodes successfully in the target muscle or because some subjects were part of a different experiment (S5-S7). For all subjects, except S3 and S6, data were analysed from two intrinsic laryngeal EMG channels and either orbicularis oris or levator labii. In the case of S3, two orofacial muscle channels (L-OOI and R-LLS) and one laryngeal channel (LCT) were analysed. For S6, the EMG of a lip muscle was not available, and the activity of medial pterygoid, a jaw closing muscle, was analysed along with that of TA and CT.

For normal speakers, the 20 one second FLU data files were taken randomly from the large samples of fluent speech recorded during the speech and reading tasks. The only criterion for selection of these intervals was one that was applied to all intervals selected for all tasks: that all EMG channels appear to have some activity during the interval. The presence of EMG activity was verified by visual inspection of the records or, in questionable cases, by a computer program that determined if the voltage was above an "activation threshold" for at least $25 \%$ of the record. ${ }^{6}$ This criterion was applied because work from our laboratory has shown that spectral analysis of records with no EMG activity can produce spurious spectral peaks and cross channel correlations owing to the presence of noise that is common across channels.

Selection of the FLU data for the stuttering subjects was more problematic. Files were selected that (1) contained 1 second of speech that two observers agreed had no perceptible stuttering, and (2) were not immediately adjacent (within 0.5 second) to a disfluent interval. Analyses of FLU speech were not completed for four of the stuttering subjects. These subjects had such severe stuttering that it was not possible to collect enough 1 second intervals for analysis within the above criteria. For each of the stuttering subjects whose fluent data were analysed (S2, S3, S5), 20 FLU files could not be obtained, and the analysis was completed with fewer (at least nine) files.

For the STUT data, 1 second intervals that contained a disfluency were selected. These 1 second STUT intervals could be part of a longer disfluent event, or they could contain a disfluency with a duration $<1$ second. Because earlier work has shown that type of disfluency based on perceptual linguistic categories-for example, sound repetition, sound prolongation, interjection, is not related to the presence of oscillatory EMG activity, all types of disfluencies were included. Two judges listened to the audiotape for the selected intervals of perceptually FLU and STUT speech, and in the case of a disagreement, the interval was not included in the analysis. There was no consistent pattern of disfluency observed across the various speech conditions for the stuttering subjects. Some were more disfluent on the reading tasks, while others experienced more difficulty in conversational speech.

One second intervals for the pitch glide and sustained vowel tasks were selected randomly throughout the subjects' repeated performances of each task. Only the laryngeal EMGs were analysed for these tasks, which often did not involve significant activation of orofacial muscles. Subjects S5-S7 did not perform these tasks.

After digitisation, the 1 second data files were full wave rectified and smoothed with a 10.25 ms gaussian window. After this processing, the power spectrum of each 1 second record was computed (for details of the power spectrum computation see Smith and Denny $^{18}$, and these spectra were averaged for each muscle, subject, and task.

In addition, the coherence function was computed for within subject pairs of EMG signals. Coherence ranges from $0-1 \cdot 0$ and is equivalent to the squared cross correlation between power in two signals computed separately for each frequency. ${ }^{19}$ If the power at a particular frequency covaries over the 20 one second samples of two EMG signals for the task, the coherence between the two signals will be a significant, non-zero value at that frequency.

Averaged power spectra and coherence functions were plotted for each subject and task. The locations of spectral maxima were determined, and frequency of occurrence of spectral maxima within three ranges, $0-4 \mathrm{~Hz}$, $5-15 \mathrm{~Hz}$, and $>15 \mathrm{~Hz}$, was tabulated. The occurrence of significant values of coherence within the $5-15 \mathrm{~Hz}$ band was also noted. Coherence functions calculated on less than 16 files were not considered to be meaningful, as limited sampling of noisy signals can lead to spuriously high coherence estimates. ${ }^{19}$ For 20 files (20 seconds of sampled data) with the present sampling parameters, coherence estimates $>0.08$ are significantly different from 0 with $p<0.05$; for 16 files this value is $0 \cdot 10$. For consistency, the more conservative limit for non-zero coherence, $0 \cdot 10$, was used to evaluate all coherence functions.

\section{Results \\ SPEECH \\ Normal speakers}

Figure 1 shows the averaged spectra computed from the amplitude envelopes of the EMG for the three normal speaking subjects for all muscles. In all cases these spectra show 
Figure 1 Averaged power spectra computed from the amplitude envelopes of the EMG for the three normal speaking subjects $N 1(A)$, N2 $(B)$ and N3 (C). The 5-15 Hz band is marked by vertical lines in all spectra.
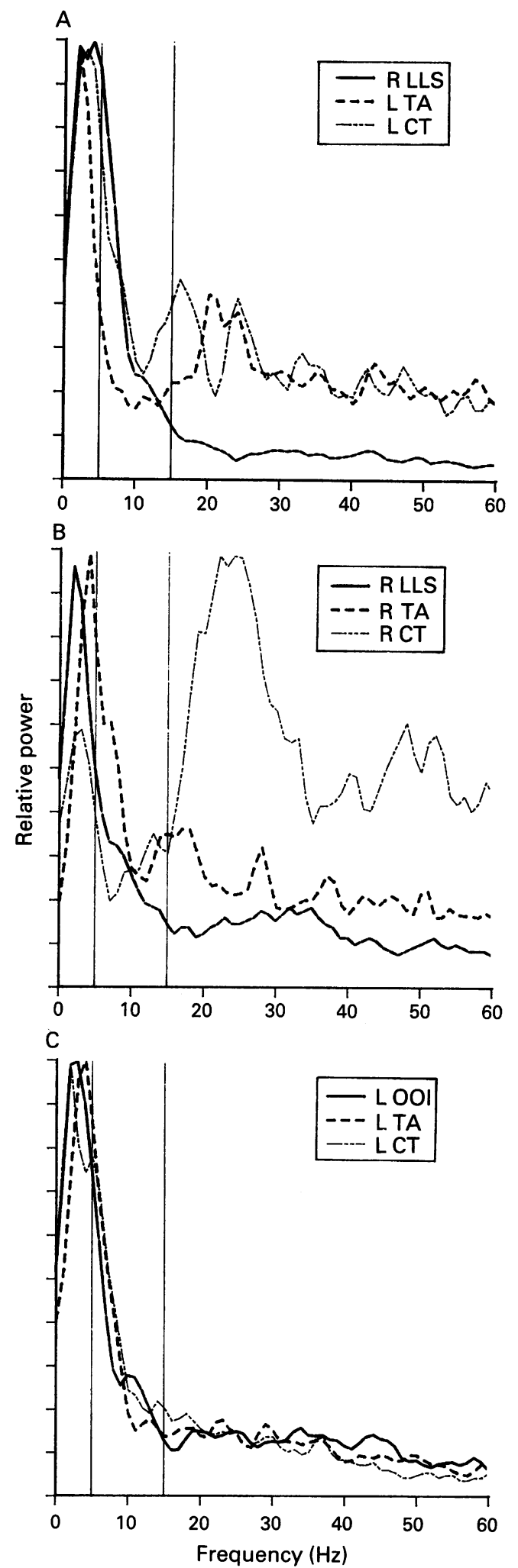

Table 1 Location (frequency) of maxima in spectra of amplitude envelopes of EMGs during speech by normal speakers.

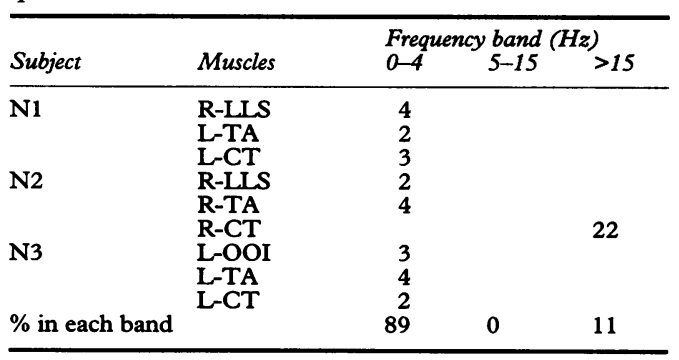

very large peaks in the $0-4 \mathrm{~Hz}$ range, suggesting that during speech, the amplitude envelope of the EMG is consistently modulated in this frequency band. Summarised in table 1 are the locations of the spectral maxima for the speech condition for the normal speakers. For eight of nine spectra, the maximum value falls between 2 and $4 \mathrm{~Hz}$. No maxima were observed in the $5-15 \mathrm{~Hz}$ band; and only the CT spectrum of $\mathrm{N} 2$ shows a maximum above $15 \mathrm{~Hz}$. The coherence functions computed for the nine muscle pairs analysed in the normal subjects' speech condition revealed no instances of significant, non-zero coherence in the $5-15 \mathrm{~Hz}$ band.

\section{Stutterers}

Stuttering subjects showed a variety of patterns of laryngeal muscle activation during stuttering. Two examples are shown in figures $2 \mathrm{~A}$ and $3 \mathrm{~A}$, which contain EMG and audio records illustrating stuttered speech for S2 and S5. The records show 6 seconds of data from a typical disfluency for each subject. Figures $2 \mathrm{~B}$ and $3 \mathrm{~B}$ contain the averaged power spectra for these two subjects computed from the STUT files for all muscles.

The time domain plots of data for subject S2 (fig 2A) suggest the presence of tremorlike oscillations for both OOI and CT during stuttering. The averaged power spectra for these muscles (fig 2B) demonstrate that, during stuttering, both of these channels show a strong $11 \mathrm{~Hz}$ oscillation. Similarly, tremorlike oscillations are visually apparent in the OOS and TA channels for S5 (fig 3A), and peaks at $8 \mathrm{~Hz}$ dominate the associated averaged power spectra shown in figure 3B.

Table 2 indicates the distribution of the maxima of the spectra computed from stuttered speech intervals. Five of the seven stuttering subjects had maxima in the $5-15 \mathrm{~Hz}$ band in at least one muscle. Forty-three percent of the 21 spectra had maxima in the $0-4 \mathrm{~Hz}$ range, while $48 \%$ had maxima in the $5-15 \mathrm{~Hz}$ band, and $9 \%$ had maxima above 15 $\mathrm{Hz}$. Table 2 also contains the distribution of spectral maxima for the averaged power spectra computed from stutterers' fluent speech. Only one of these spectra (TA for S2) had a maximum in the $5-15 \mathrm{~Hz}$ band.

Analysis of three muscle pairs for each of the seven stuttering subjects yielded 21 coherence functions. The maximum value of the coherence function in the $5-15 \mathrm{~Hz}$ band for all muscle pairs for the STUT data was noted. Six of seven stuttering subjects had significant coherence in the $5-15 \mathrm{~Hz}$ band in at least one muscle pair. Significant coherence occurred both within systems (for example, TA/CT pairs) and across systems (for example, OOI/TA). Figure 4 shows coherence functions computed for the OOI/CT pair of S2 and the OOS/TA pair of S5. It was noted above that these muscle pairs showed maxima at a common frequency in the power spectra $(11 \mathrm{~Hz}$ for OOI and CT of S2, figure $2 \mathrm{~B}$, and $8 \mathrm{~Hz}$ for OOS and TA of S5, figure 3B). As figure 4 indicates, the $11 \mathrm{~Hz}$ oscillation of S2 was not coherent, but the coher- 


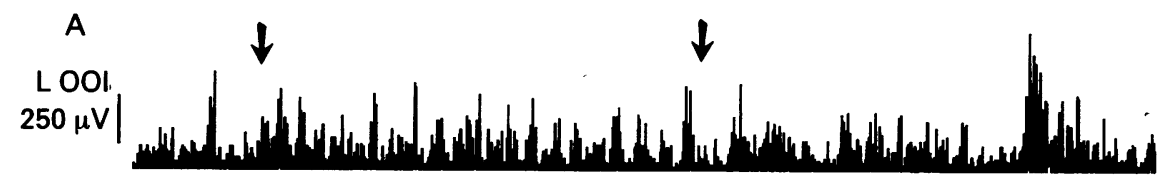

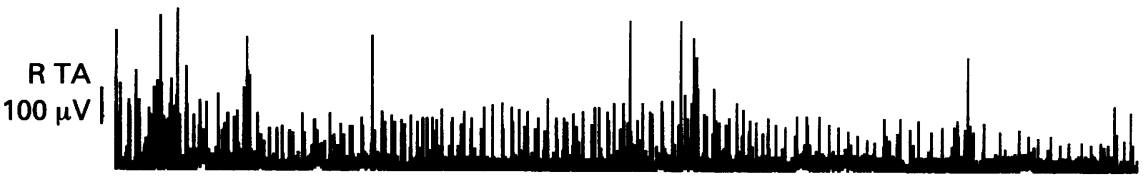
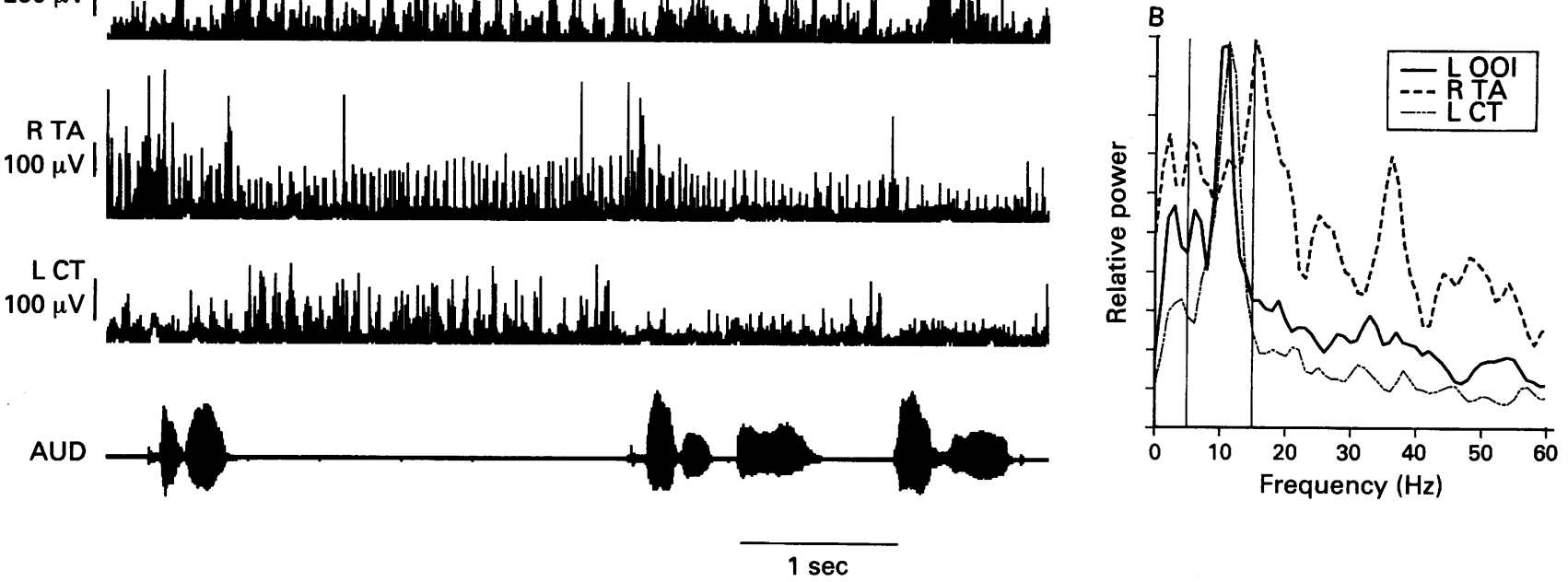

Figure 2 (A) Typical pattern of muscle activity associated with disfluent speech of subject S2. The arrows indicate the interval in which stuttering occurred. (B) Power spectra computed from amplitude envelopes of EMGs of 22 from the stuttered files.
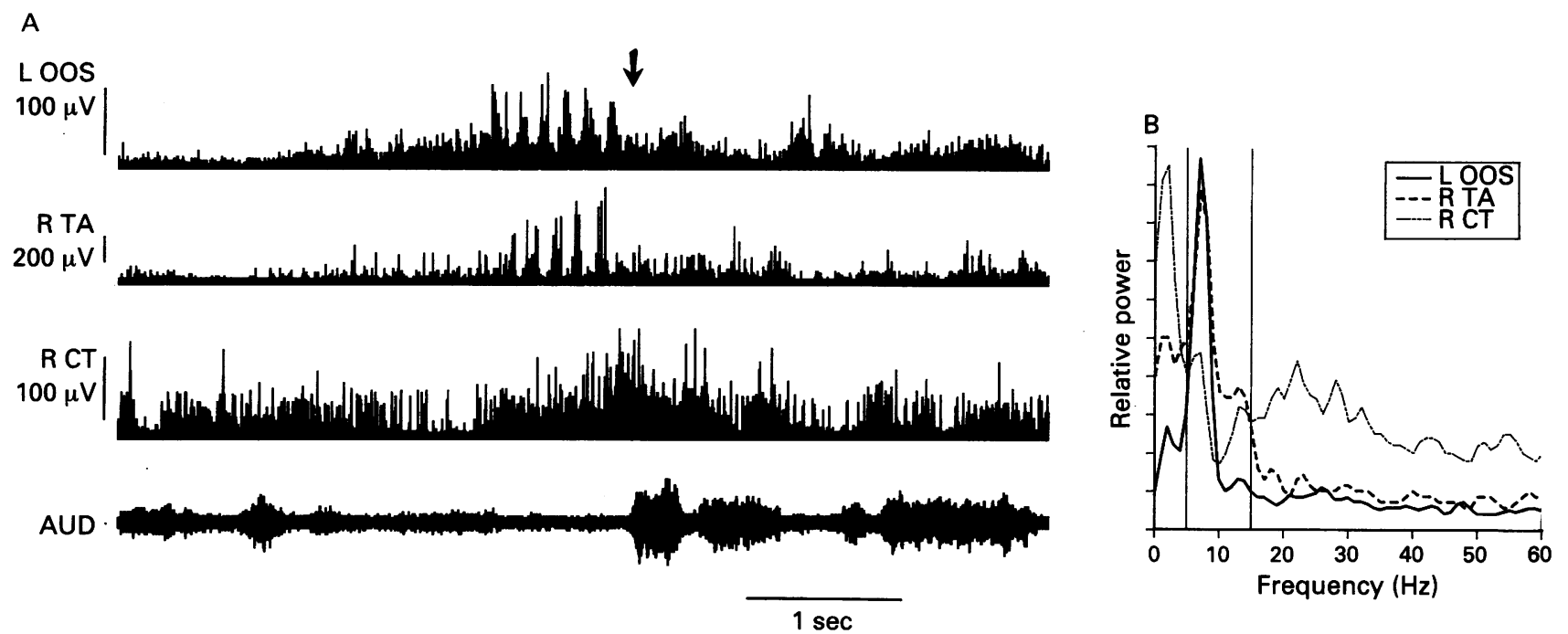

Figure 3 (A) Typical pattern of muscle activity associated with disfluent speech of subject S5. The arrow indicates the end of a long interval in which stuttering occurred. The audio recording for this subject had a high level of background noise, but the onset of fluent speech (marked by the arrow) can still be detected visually. (B) Power spectra computed from amplitude envelopes of EMGs of S5 from the stuttered files.

Table 2 Location (frequency) of maxima in spectra of amplitude envelopes of stutterers' EMGs during stuttered speech (STUT) and fluent speech (FLU)

\begin{tabular}{|c|c|c|c|c|c|c|c|}
\hline \multirow[b]{2}{*}{ Subject } & \multirow[b]{2}{*}{ Muscle } & \multicolumn{3}{|c|}{$S T U T$} & \multicolumn{3}{|l|}{$F L U$} \\
\hline & & \multicolumn{3}{|c|}{ Frequency band $(\mathrm{Hz})$} & \multicolumn{2}{|c|}{ Frequency band $(\mathrm{Hz})$} & $>15$ \\
\hline S1 & $\begin{array}{l}\text { L-OOI } \\
\text { L-TA } \\
\text { L-CT }\end{array}$ & 1 & & $\begin{array}{l}20 \\
18\end{array}$ & & & \\
\hline S2 & $\begin{array}{l}\text { L-OOI } \\
\text { R-TA }\end{array}$ & & $\begin{array}{l}11 \\
15\end{array}$ & & & 15 & \\
\hline S3 & $\begin{array}{l}\text { L-CT } \\
\text { L-OOI }\end{array}$ & 2 & 11 & & $\begin{array}{l}3 \\
2\end{array}$ & & \\
\hline & & $\begin{array}{l}3 \\
2\end{array}$ & 5 & & $\begin{array}{l}3 \\
3\end{array}$ & & \\
\hline S4 & $\begin{array}{l}\text { L-LLS } \\
\text { L-TA }\end{array}$ & $\begin{array}{l}4 \\
1\end{array}$ & & & & & \\
\hline S5 & $\begin{array}{l}\text { L-OOS } \\
\text { R-TA }\end{array}$ & & $\begin{array}{l}7 \\
7\end{array}$ & & $\begin{array}{l}2 \\
3\end{array}$ & & \\
\hline S6 & $\begin{array}{l}\text { R-CT } \\
\text { R-MP }\end{array}$ & 2 & & & 2 & & \\
\hline So & $\begin{array}{l}\text { R-CT } \\
\text { R-TA }\end{array}$ & & $\begin{array}{l}0 \\
5 \\
5\end{array}$ & & & & \\
\hline S7 & $\begin{array}{l}\text { L-OOS } \\
\text { R-TA }\end{array}$ & $\begin{array}{l}2 \\
2\end{array}$ & & & & & \\
\hline$\%$ in each band & L-TA & 43 & $\begin{array}{l}10 \\
48\end{array}$ & 9 & 89 & 11 & 0 \\
\hline
\end{tabular}

ence function of $\mathrm{S} 5$ shows that the $8 \mathrm{~Hz}$ oscillations of OOS and TA were highly correlated.

Coherence analyses of stutterers' fluent speech were completed only for S3, who had enough files (19) for the coherence estimate. These functions revealed no significant coherence in the $5-15 \mathrm{~Hz}$ band for any muscle pair.

PITCH GLIDES AND SUSTANNED “EE” (N1-N3 AND S1-S4)

Table 3 indicates the location of the spectral maxima for the pitch glides and sustained "ee" performed by the normal speakers and stutterers S1-S4. For both groups the most common spectral pattern was one with a maximum above $15 \mathrm{~Hz}$. This pattern is illustrated for the pitch glides of $\mathrm{N} 1$ and $S 1$ in figure $5 \mathrm{~A}$ and $\mathrm{B}$. As shown in table 3, maxima in the $5-15 \mathrm{~Hz}$ band did emerge for one mus- 


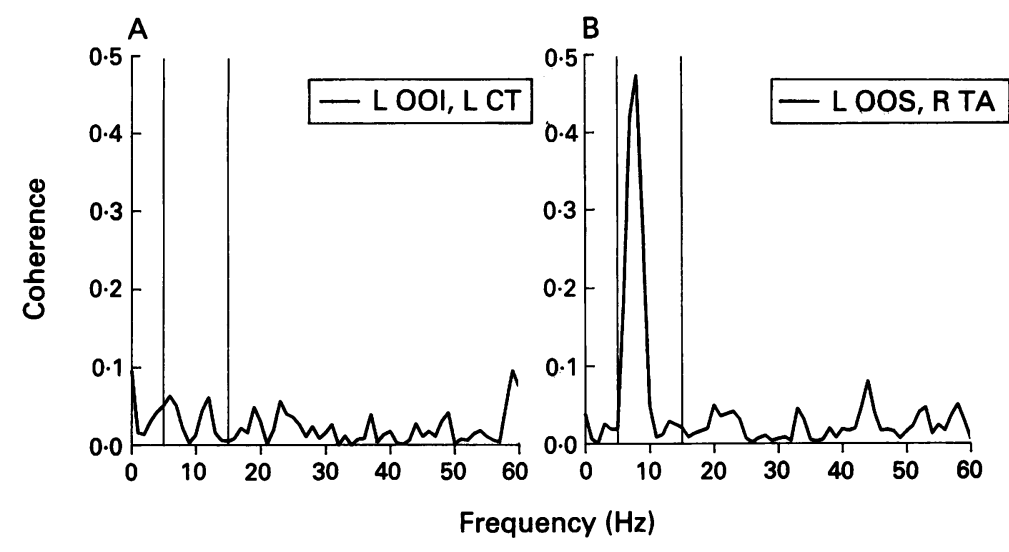

Figure 4 (A) Coherence function computed for the L OOI and LCT pair of S2 on the basis of the stuttered data. (B) Coherence function computed for the L OOS and RTA pair of $S 5$ on the basis of the stuttered data.

cle for three subjects $(\mathrm{N} 2, \mathrm{~N} 3, \mathrm{~S} 2)$ in the sustained "ee" task. The CT and TA spectra for $\mathrm{N} 2$ for the sustained "ee" are shown in figure $5 \mathrm{C}$.

Significant, non-zero coherence in the 5-15 $\mathrm{Hz}$ band was observed in two subjects in the pitch glide condition $(\mathrm{N} 1$, coherence $=0 \cdot 11$ at $11 \mathrm{~Hz}$; S4, coherence $=0.21$ at $6 \mathrm{~Hz}$ ) and in two subjects for the sustained "ee" condition $(\mathrm{N} 1$, coherence $=0.12$ at $15 \mathrm{~Hz} ; \mathrm{S} 2$, coherence $=0.20$ at $6 \mathrm{~Hz}$ ).

\section{Discussion}

NORMAL SPECTRAL PATTERNS

Because earlier investigations have not computed spectra of the EMG amplitude envelopes of laryngeal muscle activity, it was not known what spectral patterns would characterise the activity of TA and CT during the speech of normal subjects. During speech the position and stiffness of the vocal folds must be continuously adjusted to produce voiced and unvoiced sounds and to vary the pitch of the voice. TA plays a role in vocal fold adduction/abduction and in adjusting the internal stiffness of the vocal fold. ${ }^{20} \mathrm{CT}$ provides the major mechanism for adjusting the length of the vocal folds and thus is the muscle primarily responsible for changing the rate of vocal fold vibration. ${ }^{20}$

Table 3 Location (frequency) of spectral maxima for power spectra computed for pitch glides and sustained "ee"

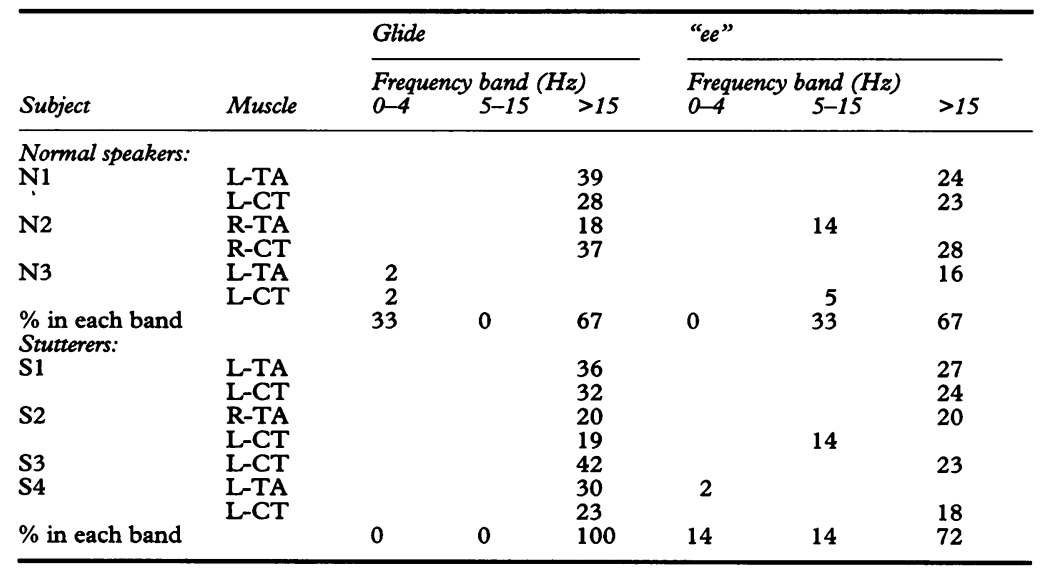
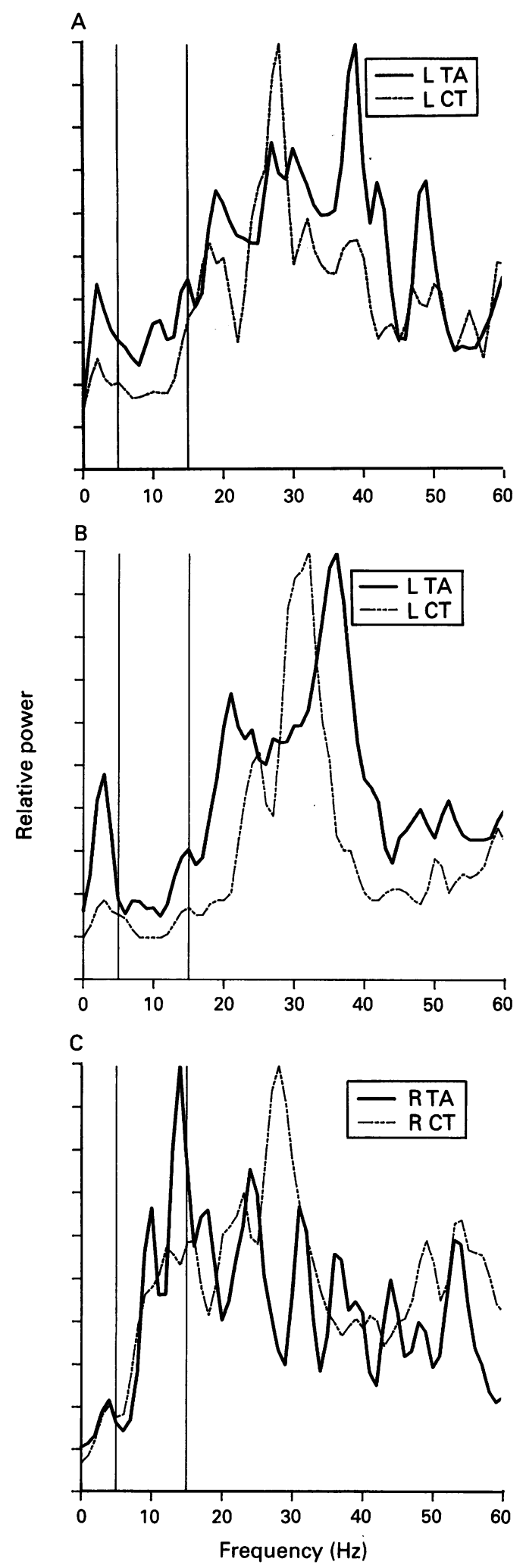

Figure 5 Power spectra computed from amplitude envelopes of laryngeal EMGs recorded during pitch glides of $N 1(A)$ and $S 1$ (B), and during sustained "ee" of N2 (C).

Data recorded from the normal speakers in the present experiment clearly indicated that TA and CT activity was consistently modulated in relation to speech gestures. The dominant rate of modulation, $2-4 \mathrm{~Hz}$, appeared as the maximum value of the spectrum in most cases (fig 1A). Such spectral patterns 
are identical to those computed for the amplitude envelopes of orofacial muscle activity during normal speech. ${ }^{6}$ The rate of modulation probably reflects the rate of production of primary speech gestures, and thus the rate of syllable production.

For subject N2, although there was a clear peak in the spectrum at $3 \mathrm{~Hz}$ (fig 1B), the maximum of the spectrum occurred at $22 \mathrm{~Hz}$. Spectra with maxima above $15 \mathrm{~Hz}$ have not been observed in the amplitude envelopes of orofacial muscles recorded during normal speech in the present or in a previous experiment. ${ }^{6}$ It seems likely that these relatively high frequency maxima emerge in the laryngeal EMG envelopes when the recording is dominated by the firing of one or a few large motor unit spikes. In earlier studies in which the spectrum of the EMG amplitude envelope has been computed in speech, ${ }^{611}$ orofacial recordings have been obtained with broadfield surface or intramuscular electrodes (each wire inserted with a separate needle). The resultant EMG was a gross interference pattern in which spikes of single units typically were not distinguishable. In contrast, the bipolar laryngeal EMG electrodes are inserted with a single needle and are likely to record from a much smaller population of motor units. Preliminary analyses of motor unit firing rates in the present data indicate that rates of 20-24 spikes/s are typical.

Spectra with maxima $>15 \mathrm{~Hz}$ were the most common spectral pattern observed both for stutterers and normal speakers during performance of the singing and sustained vowel tasks. Thus, spectra for these tasks, unlike speech, are not normally dominated by a $0-4$ $\mathrm{Hz}$ modulation of the EMG. For these tasks, spectra with maxima in the $5-15 \mathrm{~Hz}$ band were rare, with only three subjects showing such spectral patterns for one muscle in the sustained vowel condition.

OSCILLATIONS IN THE 5-15 HZ BAND IN THE STUTTERED AND FLUENT SPEECH OF STUTTERERS

The majority (5/7) of the stuttering subjects had spectral maxima in the $5-15 \mathrm{~Hz}$ band in at least one muscle during disfluent speech. Maxima in this frequency band were observed for both orofacial and laryngeal muscles. The results of the present investigation thus extend the findings of earlier studies of orofacial muscle systems and lead to the conclusion that excessive oscillatory EMG activity can be present in laryngeal muscles during stuttering. It seems clear that patterns of muscle contraction necessary for normally coordinated speech movements would be disrupted by the synchronised firing of groups of motor units at rates of $5-15 \mathrm{~Hz}$.

Also consistent with earlier investigations is the finding that not all stutterers show such oscillations. ${ }^{611}$ Dominant oscillations in the 5-15 $\mathrm{Hz}$ band appear to be part of a neuromuscular pattern that characterises stuttering, ${ }^{811}$ but they are not a necessary condition for stuttering. Disfluencies clearly can occur in the absence of excessive oscilla- tions; thus other aberrant patterns of neuromuscular activity also must contribute to the breakdowns in speech production that characterise stuttering.

The fact that mild stutterers were excluded from the experiment might be interpreted to suggest that mild stutterers do not show tremor-like oscillations of EMG activity during their disfluent speech. This is not the case; earlier studies ${ }^{6}$ have demonstrated tremor-like oscillations in EMGs of mild stutterers. Mild stutterers were excluded from the present experiment because they may exhibit little stuttering in a single, 2 hour session. Because the central goal of the experiment was to analyse EMG during disfluent speech, we believed that stutterers should not be subjected to the present invasive methods unless it seemed highly likely that they would actually be disfluent during the recording session.

Given the severe nature of the stuttering exhibited by most of the subjects tested in the present experiment, it was difficult to obtain enough tokens of perceptually fluent speech for analysis. In the three stuttering subjects for whom power spectra were computed for fluent speech, the data suggest that oscillations in the $5-15 \mathrm{~Hz}$ band were reduced or absent during fluent speech. For example, S5 had spectral maxima at $7 \mathrm{~Hz}$ in both OOS and CT during stuttering, but in fluent speech, spectral maxima for these muscles were at 2 and $3 \mathrm{~Hz}$, respectively.

Oscillations in the 5-15 $\mathrm{Hz}$ band did emerge in the sustained vowel condition for two normal speakers and one stutterer. This may reflect the appearance of tremor during a task that required subjects to sustain phonation as long as they could. In any case, the fact that two normal speakers and one stutterer showed dominant oscillations in the 5-15 $\mathrm{Hz}$ band in the sustained vowel task would suggest that this was not an aberrant feature of stutterers' vocal motor behaviour.

CORRELATIONS IN ACTIVITY ACROSS MUSCLES The present results demonstrate that oscillations in the 5-15 Hz band occur in laryngeal muscles as well as in orofacial muscle activity during stuttering. If a common source were driving these oscillations, the frequencies of oscillation should be the same, and the oscillations should covary in amplitude over time. As indicated in table 2 , in the three subjects who had oscillations in more than one muscle, spectral maxima occurred at the same frequency for at least two muscles, both within system (S6, CT and TA at $5 \mathrm{~Hz}$ ) and across systems (S2, OOI and CT at $11 \mathrm{~Hz}$ and $\mathrm{S} 5, \mathrm{OOS}$ and TA at $7 \mathrm{~Hz}$ ). The existence of a common frequency of oscillation suggests a common driving source, but the coherence function provides a better test of a hypothetical common source. The coherence functions revealed that oscillations at a common frequency could be correlated or uncorrelated. The OOI and CT oscillations of S2 at $11 \mathrm{~Hz}$ showed zero coherence. In contrast, the $7 \mathrm{~Hz}$, across system oscillations of OOS and TA of S5 were highly correlated (coherence $=0.42$ ), 
and the within system (TA and CT) oscillations of S6 showed the highest coherence value observed $(0.83)$. These mixed results are consistent with what has been observed across subjects with recordings of various orofacial muscles. ${ }^{61121}$

\section{POTENTIAL MECHANISMS UNDERLYING NEUROMUSCULAR OSCILLATIONS IN STUTTERING}

Oscillations of muscle activity are common in both normal and pathological motor systems, and there are a large number of mechanisms that can produce neuromuscular oscillations. ${ }^{9}$ At the present time, we have no data that would allow us to pinpoint with any degree of certainty the source of the neuromuscular oscillations that can characterise breakdowns in stutterers' speech. Certain aspects of the data, however, do provide a foundation for speculation about putative mechanisms.

Usually hypotheses about the source of neuromuscular oscillations attempt to distinguish the contribution of central and peripheral mechanisms. A strong central hypothesis to account for neuromuscular oscillations in stuttering would assert that a single source within the CNS generates the oscillations seen in various muscles within and across speech subsystems. The present data do not support a strong central hypothesis to account for oscillations across systems and subjects. In some cases, subjects had oscillations at different frequencies in different muscles. In other cases, oscillations could occur at a common frequency, but the oscillations were not correlated. A subject could, however, have oscillations in orofacial and laryngeal muscles that were highly correlated. Such results are consistent with the hypothesis that independent mechanisms drive oscillations in different muscles, but that these mechanisms can become entrained.

How does the oscillatory activity of a pool of motor neurons in the vagal motor nucleus become entrained with the oscillations of a pool of motor neurons in the facial nucleus? In addition to the neural systems that produce speech movements, the laryngeal and orofacial muscles are the targets of the output pathways of many other neural control systems. These include the systems involved in metabolic breathing, mastication, deglutition, and emotional expression. One possibility is that autonomic systems-for example, those involved in emotional expression-may provide a mechanism whereby oscillations can become linked across orofacial, laryngeal, and respiratory systems. With increased autonomic arousal, the neural centres involved in emotional expression may provide a positive feedback network to distributed motor neuron pools such that small, independent oscillations grow larger and ultimately become entrained.

Under this hypothesis, stuttering should be worse under conditions of increased autonomic arousal, and increased neuromuscular oscillations should be correlated with increases in autonomic arousal. On the basis of clinical evidence and subject reports, it is widely accepted that stuttering becomes worse under conditions of emotional stress. ${ }^{2}$ In addition, in a study of 19 stuttering subjects, Weber and $S$ mith $^{22}$ reported that the likelihood of disfluency and the severity of disfluency were positively correlated with the level of sympathetic arousal. Future work will be necessary to determine if the amplitude of neuromuscular oscillations in stuttering is correlated with levels of sympathetic arousal. Relevant to this argument is the demonstration of an increase in tremor in limbs of human subjects in response to increased levels of circulating adrenaline. ${ }^{23}$

\section{CONCLUSION}

Stuttering is a complex movement disorder affecting speech motor systems. The present investigation extends earlier work by demonstrating that orofacial and laryngeal muscle systems can be disturbed in common ways in stuttering. As a final caveat, it is noted that the pattern of neuromuscular oscillations described in this experiment is only a part of the constellation of symptoms that constitute the disorder of stuttering. In no way is it implied that these oscillations are the cause of stuttering (see Smith $^{1}$ for a detailed discussion of this issue). Rather it is suggested that these oscillations are a common physiological correlate of stuttering, one that may ultimately provide a partial key to understanding the neural mechanisms that disrupt speech movement patterns in this disorder.

This work was supported by grants DC00559 and DC00976 from the NIH National Institute on Deafness and Other Communication Disorders.

1 Smith A. Factors in the etiology of stuttering. $A S H A$ Reports 1990;18:39-47.

2 Van Riper C. The nature of stuttering. Englewood Cliffs: Prentice-Hall, 1982.

3 Zimmermann G. Stuttering: a disorder of movement. $g$ Speech Hearing Res 1980;23:122-36.

4 Fibiger S. Stuttering explained as a physiological tremor. Speech Transmission Laboratory-Quarterly Progress and Status Report 1971;2-3:1-24.

5 McClean M, Goldsmith H, Cerf A. Lower-lip EMG and displacement during bilabial disfluencies in adult stutterers. $\mathcal{F}$ Speech Hearing Res 1984;27:342-9.

6 Smith A. Neural drive to muscles in stuttering. 7 Speech Hearing Res 1989;32:252-64.

7 McFarland DH, Smith A, Moore CA, Weber CM Relationship between amplitude of tremor and reflex responses of the human jaw-closing system. Brain Res 1986;366:272-8.

8 Palla S, Ash MM Jr. Frequency analysis of human jaw tremor at rest. Arch Oral Biol 1979;24:709-18.

9 Stein RB, Lee RG. Tremor and clonus. In: Brookhart JM Mountcastle VB, eds. Handbook of physiology: the nervous Mountcastle VB, eds. Handbook of physiology: the nervous system II. Bethe

10 Phillippbar SA, Robin DA, Luschei ES. Limb, jaw, and vocal tremor in parkinson's patients. In: Yorkston $\mathrm{KL}$ Buekelman DR, eds. Recent Advances in Clinical Dysarthria. Boston: College-Hill, 1989:166-97.

11 Denny M, Smith A. Gradations in a pattern of neuromuscular activity associated with stuttering. $\mathcal{f}$ Speech Hearing Res 1992;35:1216-29.

12 Freeman F, Ushijima T. Laryngeal muscle activity during stuttering. $\Im$ Speech Hearing Res 1978;21:538-62.

13 Shapiro AI. An electromyographic analysis of the fluent and dysfluent utterances of several types of stutterers. f Fluency Disorders 1980;5:203-32.

14 Elble RJ, Randall JE. Motor-unit activity responsible for 8- to $12-\mathrm{Hz}$ component of human physiological finger 8- to 12-Hz component of human physi

15 Riley G. Stuttering severity instrument for children and adults. 
Portland: C.C. Publications, 1984.

16 Hirano M. Clinical examination of voice. In: Arnold GE, Winckel F, Wyke BD, eds. Disorders of human communication 5. New York: Springer-Verlag, 1981.

17 Barlow SM, Cole KJ, Abbs JH. A new head-mounted lipjaw movement transduction system for the study of motor speech disorders. $₹$ Speech Hearing Res 1983;26: 283-8.

18 Smith A, Denny M. High frequency oscillations as indicators of neural control mechanisms in human respiration, mastication, and speech. $f$ Neurophysiol 1990;63: 745-58.

19 Bendat JS, Piersol AG. Random data: analysis and measure- ment procedures. 2nd edn. New York: Wiley, 1986.

20 Hirano M, Kakita Y. Cover-body theory of vocal fold vibration. In: Daniloff RG, ed. Speech science: recent advances. San Diego: College Hill, 1985:1-46.

21 Smith $A$, Denny $M$, Wood J. Instability in speech muscle systems in stuttering. In: Peters HFM, Hulstijn W, systems in stuttering. In: Peters HFM, Hulstijn W, Starkweather W, eds. Speech motor

22 Weber CM, Smith A. Autonomic correlates of stuttering and speech assessed in a range of experimental tasks. $\mathcal{F}$ Speech Hearing Res 1990;33:690-706.

23 Marsden CD, Owen DA. The effect of adrenaline on physiological tremor in man. $\mathcal{F}$ Physiol 1967;188:40-1.

\section{Samuel Johnson: victim of Gilles de la Tourette syndrome}

The well known tics, mannerisms, postures and verba repetitions displayed by Samuel Johnson the great scholar-lexicographer suggest that he was a victim of Gilles de la Tourette syndrome. ${ }^{1-3}$

Thomas Tyers" commenting on Johnson said "he was to the last a convulsionary ... His gestures, which were a degree of St Vitus' Dance, in the street attracted the notice of many".

Boswell 156 observed "Dr Johnson is often muttering pious ejaculations, when he appears to be talking to himself."

"He commonly held his head on one side towards his right shoulder, and shook it in a tremulous manner, moving his body backwards and forwards, and rubbing his left knee in the same direction, with the palm of his hand. In the intervals of articulating he made various sounds with his mouth, sometimes as if ruminating, sometimes giving a half-whistle, sometimes making his tongue play backwards from the roof of his mouth, as if clucking like a hen, and sometimes .... pronouncing quickly under his breath, 'too, too, too'."
“... he had another particularity . . . his anxious care to go in and out at a door or passage, by a certain number of steps from a certain point . . . I have on innumerable occasions, observed him suddenly stop, and then seem to count his steps with a deep earnestness; . ..."

J M S PEARCE

1 McHenry L. Samuel Johnson's tics and gesticulations. f Hist Med 1967;22:152-68.

2 Murray TJ. Dr Samuel Johnson's movement disorder. $B M \Im$ 1979;1:1610-4.

3 Pearce JMS. Doctor Samuel Johnson: "the Great Convulsionary" a victim of Gilles de la Tourette's syndrome. $¥ R$ Soc Med 1993 (in press)

4 Tyers Thomas. A Biographical sketch of Dr Samuel Johnson. Gentleman's magazine, December 1784 (reproduced In: Hill GB. Johnsonian Miscellanies). London: duced In: Hill GB. Johnso

5 Boswell James. The life of Samuel fohnson LLD. London: James Blackwood, 1791:135-6.

6 Boswell James. The journal of $a$ tour of the Hebrides with Samuel fohnson LLD. London 1785. London: Everyman, 1948:8. 